\title{
Identification and Expression Patterns of Chemosensory Genes in Male and Female Wax Moths, Galleria mellonella
}

\author{
Shuang Yang ${ }^{1,2}$, Huiting Zhao ${ }^{3}$, Xuewen Zhang ${ }^{2}$, Kai Xu' ${ }^{1}$, Lina Guo ${ }^{1}$, Yali Du ${ }^{1}$ \\ and Yusuo Jiang,* \\ ${ }^{1}$ College of Animal Science, Shanxi Agricultural University, Taigu 030801, P.R. China \\ ${ }^{2}$ Institute of Sericulture and Apiculture, Yunnan Academy of Agricultural Sciences, \\ Mengzi 661101, P.R. China \\ ${ }^{3}$ College of Life Sciences, Shanxi Agricultural University, Taigu 030801, P.R. China
}

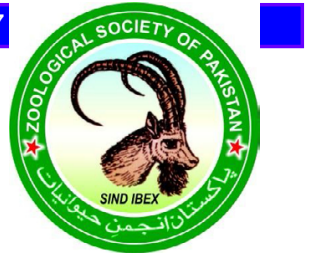

Article Information

Revised 09 December 2020

Accepted 12 March 2021

Available online 07 July 2021

Authors' Contribution

SY and YJ conceptualized the study and defined methodology. SY, KX,

LG and YD performed data curation.

SY and $\mathrm{HZ}$ analyzed the data. SY

acquired funds for the study. SY

wrote the manuscript. $\mathrm{HZ}, \mathrm{XZ}$ and

YJ reviewed the manuscript. YJ

supervised the study.

Key words

Antenna, Chemosensory gene,

Expression profile, Galleria mellonella,

Phylogenetic analysis, Wax moth,

Olfactory protein, Odorant-binding

protein, Ionotropic receptor,

Sensory neuron membrane protein,

Transcriptome analysis.

\section{INTRODUCTION}

$\mathrm{T}$ he greater wax moth, Galleria mellonella (Linnaeus, 1758), is one of the most notorious pests of honey bee colonies (Kwadha et al., 2017; Svensson et al., 2014; Dweck et al., 2010). Its larva burrows into the edges of unsealed cells, and feeds on pollen, honey, beeswax and bee brood. The adult wax moth can cause galleriasis by constructing silk-lined tunnels through cell walls and on the comb surface, thus resulting in a significant loss to the beekeeping industry (Bombelli et al., 2017; Kwadha et al., 2017; Krams et al., 2015; Svensson et al., 2014). Besides, an infestation of honey bee colonies by $G$. mellonella larvae often leads to colony losses, absconding and smallscale migration (Ellis et al., 2013; Kwadha et al., 2017). As a result, $G$. mellonella-infested honey bee combs in hives exerts a serious side effect on bees (Svensson et $a l ., 2014)$. Recently, the black queen cell virus and Israeli acute paralysis virus were found in $G$. mellonella larvae, and the spores of Paenibacillus spp. were also detected in

\footnotetext{
* Corresponding author: jiangys-001@163.com

0030-9923/2021/0005-1767 \$ 9.00/0

Copyright 2021 Zoological Society of Pakistan
}

fecal pellets of the larvae (Kwadha et al., 2017; Traiyasut et al., 2016; Hood et al., 2003; Charriere and Imdorf, 1999). This indicates that both G. mellonella adults and larvae are potential vectors of honey bee disease-causing pathogens. The damage caused by this moth is believed to contribute to the substantial decreases in both honey and native honey bee populations (Kwadha et al., 2017; Strauss et al., 2013). Given the growing concern about the health of honey bees and the economic downfall caused by $G$. mellonella infestation of honey bee colonies, it is necessary to develop alternative strategies for controlling this moth

As a nocturnal species, freshly eclosed wax moths often fly away from the bee hives to mate at night. After mating, the gravid females re-enter the hives and lay eggs in small cracks or crevices (Kwadha et al., 2017). Upon hatching, G. mellonella larvae move into the bee combs where they begin to feed and, ultimately, damage the comb (Kwadha et al., 2017; Ellis et al., 2013). Therefore, controlling the mating process and egg-laying behaviors of G. mellonella may be a critical strategy to reduce the damage caused by this insect. Unlike most moths, G. mellonella has distinct reproductive behavior where the males secret sex pheromones to attract potential mates (Kwadha et al., 
2017; Svensson et al., 2014; Han et al., 2003). Over the past decades, there has been a tremendous advancement in the understanding of insect chemical ecology following the development of pheromone-based trapping systems (Zhang et al., 2015a; Vogt and Riddiford, 1981), which provides an opportunity to develop convenience, costeffective and sustainable techniques for pest control.

G. mellonella uses a fined-tuned olfactory system located in the sensory hairs (sensilla) on each antenna to detect pheromones and other odors (Grosjean et al., 2011). A diverse range of olfactory proteins, such as chemosensory protein (CSP), ionotropic receptor (IR), odorant-binding protein (OBP), olfactory receptor (OR) and sensory neuron membrane protein (SNMP), are highly expressed in the sensillum lymph (Zhao et al., 2016; He et al., 2015; Sanchez-Gracia et al., 2009). OBPs comprise 6 cysteine residues that form 3 disulfide bonds, thereby generating a hydrophilic pocket that binds to volatile compounds (Pelosi et al., 2014). CSPs are another class of soluble binding proteins enriched in the sensillum lymph, and are consisted of 4 conserved cysteines that form 2 disulfide bonds (Vieira and Rozas, 2011). CSPs are involved in the process of semiochemical detection, and can be found in the chemosensory/non-chemosensory organs (Zhao et al., 2016; Liu et al., 2014; Gu et al., 2012). ORs, seven-transmembrane proteins, can respond to odors and pheromones through coexpression with a conserved co-receptor (Orco) and subsequently trigger signal transduction pathways by converting the chemical signals of the active odor molecules into electrophysiological signals (Cao et al., 2014; Benton et al., 2009). IRs are associated with ionotropic glutamate receptors (iGluRs) and responsible for the recognition of ammonia and acids (Rogers et al., 2001). These reporters have been found in some insect species from different orders (Cao et al., 2014), but have only been comprehensively assessed in Drosophila melanogaster. SNMPs, which contain two orthologs of SNMP1 and SNMP2, can facilitate the ligand delivery to receptors (Rogers et al., 2001). SNMP1 is specifically expressed in the pheromone-responsive olfactory receptor neurons of $D$. melanogaster, and is responsible for mediating the sensitivity of these neurons to cis-vaccenyl acetate stimulation (He et al., 2019; Cao et al., 2014; Liu et al., 2012). Thus, identification of these chemosensory genes in G. mellonella can help to improve the current moth trapping systems.

In the past few years, antennal transcriptome sequencing has been successfully employed to detect a number of candidate olfactory genes in lepidopterans, including Bombyx mori (Fang et al., 2015), Chilo suppressalis (Cao et al., 2014), Sesamia inferens (Zhang et al., 2014), Loxostege sticticalis (Wei et al., 2017) and Mythimna separata (Chang et al., 2017). Besides, several other moth species, such as the hymenopteran Apis cerana, hemipteran Adelphocoris suturalis and dipteran Bactrocera dorsalis have been sequenced by Zhao et al. (2016), Cui et al. (2017) and Jin et al. (2017), respectively. More recently, Zhao et al. (2019) performed transcriptome sequencing on G. mellonella antennae and identified several chemosensory genes (e.g., 46 ORs, 25 IRs, 22 OBPs, 20 CSPs and 2 SNMPs). Similarly, Lizana et al. (2020) also identified 20 OBP genes from this moth species. However, the number of OBPs in $G$. mellonella is relatively lesser compared to other Pyralid moths, such as C. suppressalis, Ostrinia furnacalis, and Cnaphalocrocis medinalis, with 23, 26 and 26 OBP genes, respectively (Liu et al., 2017; Zhang et al., 2015b; Cao et al., 2014). Hence, the present research aimed to examine the differential expression patterns of chemosensory genes in the antennae of female and male G. mellonella, which will serve as a guide for future works, particularly those on the prevention and/or treatment of moth infestation using male pheromones as a bait.

\section{MATERIALS AND METHODS}

\section{Sampling}

G. mellonella pupae and larvae were sampled from the infected hives in an apiary at Shanxi Agricultural University (Shanxi, China). Newly emerged adult wax moths were used to preserve the stock culture. All $G$. mellonella larvae were reared on old honeycombs in an incubator at $34 \pm 1{ }^{\circ} \mathrm{C}$ with $65 \pm 5 \%$ relative humidity in constant darkness. For transcriptomic sequencing, approximately 100 antennae per sex were collected from freshly emerged female and male moths (three replicates per sex). All samples were quickly frozen in liquid nitrogen and then kept at $-80^{\circ} \mathrm{C}$ for later use.

\section{RNA isolation, cDNA library construction and RNA-seq}

Total RNA was isolated from the antennae using TRIzol $^{\mathrm{TM}}$ Reagent (Invitrogen, CA, USA) in compliance with the manufacturer's instructions. The residual DNA was removed using DNase I (Promega, WI, USA). RNA quality was assessed using a $1 \%$ agarose gel dissolved in electrophoresis buffer. RNA-Seq library preparation and Illumina sequencing were carried out by Novogene Bioinformatic Technology (Beijing, China).

Raw data in FASTQ format were processed by PERL scripts built in house. After eliminating the low-quality reads and those with adapter sequences or poly-N, clean sequences were retained. Next, the sequence duplication level as well as the Q20, Q30, and GC content of the highquality reads were determined. Finally, clean data were 
assembled into unigenes using Trinity software (r201404-13p1; Trinity Software Solutions Inc., Waterford, VA, USA) (Grabherr et al., 2011), and each unigene was assigned a unique gene ID. All downstream analyses were then performed on the high-quality sequences. The Illumina sequencing data generated in this study were submitted to the sequence read archive (SRA) of the National Center for Biotechnology Information (NCBI) (accession No. SRR11446320).

\section{Functional annotation}

The functions of new genes were analyzed by conducting a basic local alignment search tool (BLAST) search against seven databases, including Gene ontology (GO), NCBI nucleotide sequences (Nt), NCBI nonredundant protein $(\mathrm{Nr})$, Swiss-Prot, protein families (Pfam), Kyoto encyclopedia of genes and genomes orthology (KO), and euKaryotic ortholog groups (KOGs). Proteins with the highest sequence identity for a given unigene were retrieved together with their respective functional annotation categories. The open reading frames (ORFs) of the candidate chemosensory genes were analyzed by the NCBI ORFfinder. The putative N-terminal signal peptides of OBP and CSP genes were estimated using the SignalP 5.0 Server. The conserved domains of OBP and CSP genes were predicted using the NCBI Conserved Domain Database. The transmembrane domains (TMDs) of IR, OR and SNMP genes were determined through the TMHMM version 2.0 Server. Subsequently, a phylogenetic tree was built according to the amino acid sequences of putative chemosensory genes in $G$. mellonella as well as the homologous sequences found in other lepidopteran species. After aligning the amino acid sequences through ClustalW Version 2.1, an unrooted neighbor-joining tree was established by MEGA5.2, and the branch support was evaluated with 1,000 bootstrap replications (Tamura et al., 2011).

HTSeq version 0.9.1 was applied to estimate the expression levels of candidate genes (Trapnell et al., 2010). After that, the Fragments Per Kilobase per Million mapped reads (FPKM) value of each chemosensory gene was calculated based on the following equation:

$\mathrm{FPKM}=(1,000,000 \times \mathrm{C}) /(\mathrm{N} \times \mathrm{L} \times 1000)$

Where, $\mathrm{C}$ represents the number of reads uniquely aligned to the chemosensory gene, $\mathrm{N}$ denotes the total number of fragments mapped to all unigenes, and $\mathrm{L}$ indicates the number of bases in each chemosensory gene (Mortazavi et al., 2008).

RNA-Seq by Expectation-Maximization (RSEM) software was used to map the clean sequences to the transcriptomic unigenes based on the default settings $(\mathrm{Li}$ and Dewey, 2011). The differential expression between two transcriptomes (duplicate biological samples) was analyzed by DESeq Version 1.12.0 (Wang et al., 2010). Differentially expressed genes were those with an adjusted $P$-value of $<0.05$. GO enrichment and KEGG pathway analyses were conducted using the GOseq $\mathrm{R}$ package version 1.10.0 and KOBAS Version 3.0, respectively. Online tools and databases used in this study are listed in Table I.

\section{Validation of gene expression level}

Quantitative real-time polymerase chain reaction (qRT-PCR) was carried out to validate the levels of differentially expressed genes (DEGs). The specific primers were designed with Primer3.0Plus server,and elongation factor 1-alpha $(E f-1 a)$ was employed as an internal standard for data normalization (Table II). RNA extraction and cDNA synthesis were performed with $\mathrm{TRIzol}^{\mathrm{TM}}$ Reagent (Invitrogen) and PrimeScript ${ }^{\mathrm{TM}}$ RT Reagent Kit with gDNA Eraser (Takara Bio Inc., Dalian, China), respectively, by following the manufacturer's protocols. In PCR, $2 \mu \mathrm{L}$ of 1:3 diluted cDNA was used as the template, with a reaction mixture of total volume of $20 \mu \mathrm{L}$. The qRT-PCR was conducted on an Applied Biosystems $^{\mathrm{TM}} 7500$ Fast Real-Time PCR System (Applied Biosystems, CA, USA), with the following reaction conditions: denaturation for $4 \mathrm{~min}$ at $95^{\circ} \mathrm{C}$, followed by 40 cycles of $15 \mathrm{~s}$ at $95^{\circ} \mathrm{C}$ and $34 \mathrm{~s}$ at $60^{\circ} \mathrm{C}$. The specificity of the qRT-PCR reaction was assessed by performing a

Table I.- Online tools databases and used in this study.

\begin{tabular}{ll}
\hline Online tools and databases & URLs \\
\hline NCBI ORFfinder & https:/www.ncbi.nlm.nih.gov/orffinder/ \\
SignalP 5.0 Server & http:/www.cbs.dtu.dk/services/SignalP \\
NCBI Conserved Domain Database & http://www.ncbi.nlm.nih.gov/Structure/cdd/wrpsb.cgi \\
TMHMM version 2.0 Server & http://www.cbs.dtu.dk/services/TMHMM/ \\
ClustalW V2.1 & https:/www.genome.jp/tools-bin/clustalw \\
KOBAS (KEGG Orthology Based Annotation System) V3.0 & http://kobas.cbi.pku.edu.cn/ \\
Primer3.0Plus server & http://www.bioinformatics.nl/cgi-bin/primer3plus/primer3plus.cgi \\
\hline
\end{tabular}


melting curve analysis of $20 \mathrm{~s}$ at $95^{\circ} \mathrm{C}, 30 \mathrm{~s}$ at $60^{\circ} \mathrm{C}$, and 30 $\mathrm{s}$ at $95^{\circ} \mathrm{C}$. Each candidate gene had 3 biological replicates, and 3 technical replicates were tested for each biological replicate. The relative quantification of PCR results was performed using the comparative CT method $\left(2^{-\Delta \Delta C t}\right)$.

Table II.- Primers used for qRT-PCR analysis on differentially expressed genes (DEGs).

\begin{tabular}{|c|c|}
\hline Primer name & Primer sequence (5'-3') \\
\hline \multirow[t]{2}{*}{ CSP10 } & F: TGGTCATGGCCTATCCTCGG \\
\hline & R: ATAGGGCACCAAAAGGCGTC \\
\hline \multirow[t]{2}{*}{$C S P 7$} & F: GCGTTCTTGACGAAGGAAGG \\
\hline & R: GGAGCCGTTGCGTTGATT \\
\hline \multirow[t]{2}{*}{ OBP72-like } & F: GAGGAAACAGTGCCAACCCA \\
\hline & R: CAGGAGCAGGTCAGCTTGTT \\
\hline \multirow[t]{2}{*}{ OBP89a-like } & F: GTAGACTTCGGCCTGGTGGA \\
\hline & R: CCTCTCCGTCACTCATCATCC \\
\hline \multirow[t]{2}{*}{ OR29 } & F: GCAGCATATAACAGCGAATGGA \\
\hline & R: CCTTCTTGCCGATCTTGAACA \\
\hline \multirow[t]{2}{*}{ P450-1 } & F: ACTTAGAGGCATCGCGTGGT \\
\hline & R: TGGCTCGGTACACTCTCCTG \\
\hline \multirow[t]{2}{*}{$P 450-2$} & F: GGTGTACTTAATGACTCAACGTGGT \\
\hline & R: AAGGCACAAGCTGATATTGTCG \\
\hline \multirow[t]{2}{*}{ GR67 } & F: TCTGAGAGAGAGGCATACTGCTG \\
\hline & R: TTCTAACTCTTCATGCGAATCGTC \\
\hline \multirow[t]{2}{*}{$J H B P$} & F: CGGCGAACCTAAGCTCACTT \\
\hline & R: CCATAGACAGCATCCGCTACC \\
\hline \multirow[t]{2}{*}{$\operatorname{CoA}$} & F: GGCCTCGACACCAACAGATT \\
\hline & R: CCTCAGCGACCATCTTGTCA \\
\hline \multirow[t]{2}{*}{ 49-desaturase } & F: TGCTGATCCTGTGCTTCGAT \\
\hline & R: AAGCATTCCATGCCGTCTCT \\
\hline \multirow[t]{2}{*}{ Allantoicase } & F: ACTCCTCAACGGAGGCACTT \\
\hline & R: CCAATCTCCTGGCTGTCTCC \\
\hline \multirow[t]{2}{*}{ Trypsin } & F: GCACCGACGACCATAGACAA \\
\hline & R: CGCTGAATTGGAAGCAGTGT \\
\hline \multirow[t]{2}{*}{ Troponin } & F: ACACAATGGCGGATGATGAA \\
\hline & R: CCTTCTTGGCCTTGGAAGC \\
\hline \multirow[t]{2}{*}{ Cuticular protein } & F: AGCCTCATCTGGCGGTAACT \\
\hline & R: GCCGTTCTCTTCAGCGAGA \\
\hline \multirow[t]{2}{*}{ Blastopia poly- } & F: TTGCCGACTCTCTTCTGTCG \\
\hline & R: CTGTTGTATTCGCTGACATTGC \\
\hline \multirow[t]{2}{*}{$E f-1 a$} & F: CCGTGGTTATGTTGCTGGTG \\
\hline & R: TGTGGCAATCGAGTACAGGTG \\
\hline
\end{tabular}

\section{RESULTS}

Comprehensive analysis of transcriptome sequencing data Data of the antennal transcriptome of $G$. mellonella were produced using the Illumina Hiseq ${ }^{\mathrm{TM}} 2500$ platform (Illumina Inc.). In total, 135,622,632 and 134,292,198 raw reads were acquired from the libraries of female and male specimens, respectively. After eliminating adapters, ambiguous nucleotides and low-quality sequences, the female and male antennae yielded 132,262,518 and $130,841,026$ clean reads, respectively. These clean reads were assembled into 372,571 transcripts, which accounted for 39.47 gigabases with a GC percentage of $41.78 \%$ (Table III). After merging and clustering, 188,278 unigenes were obtained (mean length $=781 \mathrm{bp}$ and N50 length $=$ $1161 \mathrm{bp})$, and 107,679 unigenes ( 57\%) were $200-500 \mathrm{bp}$ in length. After annotation by tBLASTn, 108,047 (57.38\%) unigenes were allocated to more than 1 database, and 7388 $(3.92 \%)$ were allocated to all the 7 databases.

\section{Putative chemosensory gene families}

The 5 chemosensory gene groups (CSPs, IRs OBPs, ORs and SNMPs) were identified through keyword searching and manual analysis of annotated unigenes. In total, 121 chemosensory genes were annotated in the antennal transcriptome of $G$. mellonella, comprising 37 OBPs, 35 CSPs, 33 ORs, 14 IRs, and 2 SNMPs. The information on these unigenes, such as the gene ID, amino acid sequence length, ORF length and FPKM value, are provided in Table IV.

\section{Odorant-binding proteins}

Among the 37 candidate OBP genes, 31 OBPs had a full-length ORF with signal peptide prediction sequences, whereas the remaining 6 OBPs $(O B P 6, O B P 8, O B P 12$, $O B P 14, O B P 15$ and $O B P 22)$ corresponded to a partial sequence encoding 87-147 amino acids. Among the 31 full-length OBPs, $O B P 1, O B P 2, O B P 3, O B P 4, O B P 5$, OBP7, OBP9, OBP11, OBP13, OBP16, OBP17, OBP23, OBP72-like, OBP69a-like, GOBP2, GOBP3, PBP1, PBP2, $P B P 3, P B P 4, P B P 5$ and $P B P 6$ were the typical OBPs with 6 cysteines; OBP18, OBP19 and OBP56d belonged to the Minus-C OBPs without the $2^{\text {nd }}$ and $5^{\text {th }}$ cysteines; OBP10, OBP21, OBP83a-like, OBP84a and GOBP1 were the Plus-C OBPs with additional cysteine residues. Figure 1 showed the phylogenetic tree constructed based on the amino acid sequences of OBPs in $C$. medinalis, $C$. suppressalis, O. furnacalis, Conogethes punctiferalisand G. mellonella. As expected, $37 \mathrm{Gmel}$ OBPs were divided into 5 groups, namely, GOBP, PBP, typical OBPs, Plus-C OBPs and Minus-C OBPs. 


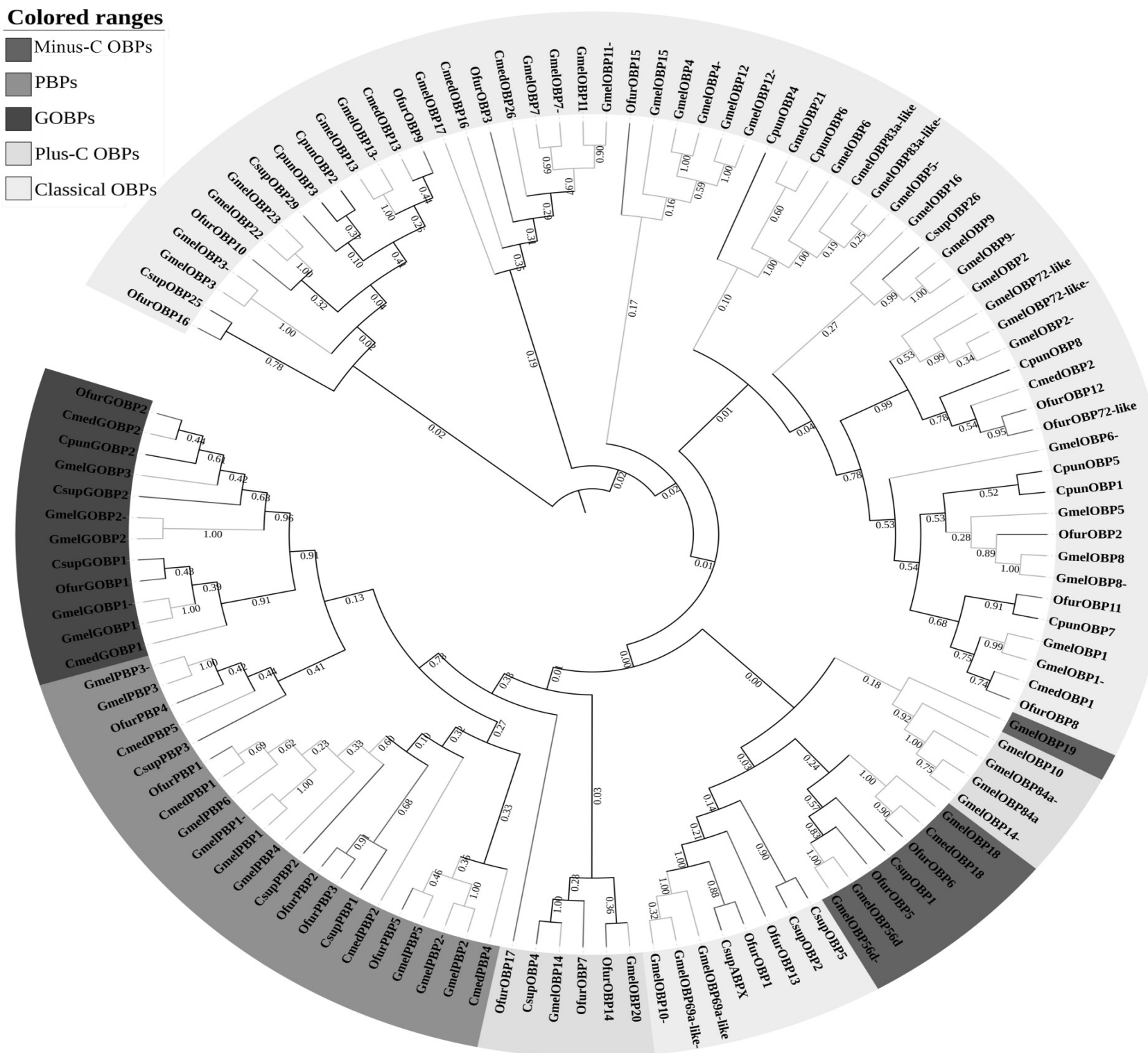

Fig. 1. Phylogenetic tree of candidate GmelOBPs with known lepidopteran odorant-binding protein (OBP) sequences. Gmel, Galleria mellonella; Csup, Chilo suppressalis; Ofur, Ostrinia furnacalis; Cmed, Cnaphalocrocis medinalis; Cpun, Conogethes punctiferalis. The clade in green and purple indicates the PBPs and GOBPs, respectively; the Minus-C OBPs subfamily is marked in red and the Plus-C OBPs subfamily is marked in blue; the Classical OBPs subfamily is marked in yellow.

Table III.- Evaluation statistical table of high quality sequencing data.

\begin{tabular}{lccccccc}
\hline Sample & Raw reads & Clean reads & Clean bases & Error (\%) & Q20 (\%) & Q30 (\%) & GC content (\%) \\
\hline C_1 & 40713720 & 39655732 & $5.95 \mathrm{G}$ & 0.02 & 97.30 & 93.07 & 41.60 \\
C_2 & 51470294 & 50320026 & $7.55 \mathrm{G}$ & 0.02 & 95.87 & 89.89 & 42.09 \\
C_3 & 43438618 & 42286760 & $6.34 \mathrm{G}$ & 0.02 & 97.27 & 92.98 & 41.37 \\
X_1 & 44502844 & 43312838 & $6.50 \mathrm{G}$ & 0.02 & 97.23 & 92.90 & 42.28 \\
X_2 & 43207642 & 42083112 & $6.31 \mathrm{G}$ & 0.02 & 97.33 & 93.14 & 42.04 \\
X_3 & 46581712 & 45445076 & $6.82 \mathrm{G}$ & 0.02 & 97.17 & 92.84 & 41.32 \\
\hline
\end{tabular}

C_1, C_2, C_3- biological replicate samples of female G. mellonella. X_1, X_2, X_3 - biological replicate samples of male G. mellonella. 
Table IV.- Annotation of candidate chemosensory genes in G. mellonella antennae.

\begin{tabular}{|c|c|c|c|c|c|c|c|c|c|}
\hline \multirow[t]{2}{*}{ Gene ID } & \multirow[t]{2}{*}{ Gene name } & \multirow{2}{*}{$\begin{array}{c}\text { Complete } \\
\text { ORF }\end{array}$} & \multirow{2}{*}{$\begin{array}{l}\text { ORF } \\
\text { (aa) }\end{array}$} & \multicolumn{6}{|c|}{ FPKM } \\
\hline & & & & C_1 & C_2 & C_3 & X_1 & X_2 & X_3 \\
\hline Cluster-99082.29367 & $C S P 1$ & Yes & 97 & 1464.71 & 789.73 & 784.16 & 1722.1 & 1513.49 & 804.82 \\
\hline Cluster-99082.26515.1 & $C S P 2$ & Yes & 126 & 2058.5 & 1278.54 & 1421.78 & 2353.67 & 1986.87 & 1767.12 \\
\hline Cluster-99082.26515.2 & CSP3 & Yes & 123 & 2058.5 & 1278.54 & 1421.78 & 2353.67 & 1986.87 & 1767.12 \\
\hline Cluster-99082.20939 & CSP4 & Yes & 125 & 634.22 & 313.09 & 420.97 & 1036.38 & 858.61 & 763.89 \\
\hline Cluster-79369.0 & CSP5 & Yes & 124 & 4.04 & 0 & 0.97 & 4.86 & 2.96 & 0.47 \\
\hline Cluster-99082.26121 & CSP6 & Yes & 131 & 20573.63 & 13120.87 & 9812.82 & 31189.81 & 30866.13 & 16460.48 \\
\hline Cluster-99082.25152 & $C S P 7 *$ & Yes & 127 & 135.9 & 101.54 & 83.43 & 284.92 & 256.77 & 250.85 \\
\hline Cluster- 48649.0 & CSP8 & Yes & 123 & 0 & 2.6 & 0 & 0 & 0 & 0 \\
\hline Cluster-99082.26231 & $\operatorname{CSP} 9$ & Yes & 147 & 22.81 & 49.18 & 65.92 & 26.2 & 20.22 & 48.45 \\
\hline Cluster-99082.6417 & CSP10* & Yes & 124 & 161.6 & 78.35 & 74.92 & 325.17 & 246.99 & 315.27 \\
\hline Cluster-99082.3437 & CSP11 & Yes & 107 & 2.46 & 1.41 & 0 & 8.13 & 0 & 9.35 \\
\hline Cluster-99082.8209 & CSP 12 & Yes & 143 & 13.29 & 0 & 13.15 & 19.92 & 13.06 & 10.56 \\
\hline Cluster-71579.0 & CSP13 & Yes & 121 & 24.05 & 20.64 & 14.52 & 32.23 & 37.13 & 20.45 \\
\hline Cluster-84608.0 & CSP14 & Yes & 121 & 0.61 & 0 & 1.76 & 1.33 & 0.58 & 0.56 \\
\hline Cluster-101784.0 & CSP 15 & Yes & 117 & 0 & 2.07 & 0 & 5.31 & 0 & 9.21 \\
\hline Cluster-99082.18933 & CSP16 & Yes & 106 & 36.39 & 0 & 36.52 & 64.54 & 38.18 & 23.88 \\
\hline Cluster-94631.0 & CSP17 & Yes & 107 & 10.51 & 11.12 & 13.2 & 13.56 & 12.85 & 9.59 \\
\hline Cluster-74631.0 & CSP18 & Yes & 107 & 21.77 & 16.48 & 26.8 & 40.22 & 37.24 & 35.71 \\
\hline Cluster-112894.0 & CSP19 & Yes & 123 & 23.45 & 5.36 & 1.55 & 40.83 & 45.84 & 26.22 \\
\hline Cluster-79965.0 & CSP20 & Yes & 129 & 13.79 & 0 & 13.99 & 24.49 & 13.25 & 11.78 \\
\hline Cluster-76369.0 & CSP21 & Yes & 126 & 0.79 & 0 & 1.14 & 2.39 & 1.32 & 0.72 \\
\hline Cluster-83402.0 & CSP22 & Yes & 129 & 3.73 & 0 & 5.03 & 8.37 & 4.39 & 2.98 \\
\hline Cluster-116429.0 & CSP23 & Yes & 120 & 0.56 & 0 & 2.71 & 3.28 & 0.55 & 1.07 \\
\hline Cluster-103453.1 & $\mathrm{CSP} 24$ & Yes & 132 & 11.5 & 0 & 7.66 & 15.5 & 7.16 & 8.04 \\
\hline Cluster-99082.7412 & CSP25 & Yes & 128 & 0 & 8.79 & 0 & 0 & 0 & 0 \\
\hline Cluster-99082.8055 & CSP26 & Yes & 128 & 43.22 & 0 & 41.01 & 67.74 & 37.86 & 29.14 \\
\hline Cluster-99082.44102 & CSP27 & Yes & 129 & 25.68 & 0 & 21.46 & 33.38 & 22.82 & 18.46 \\
\hline Cluster-99082.26548 & CSP28 & Yes & 126 & 19583.75 & 15205.67 & 22467.45 & 26367.81 & 22047.11 & 29772.2 \\
\hline Cluster-36923.0 & $\operatorname{CSP} 29$ & Yes & 126 & 0 & 45.52 & 0 & 0 & 0 & 0 \\
\hline Cluster-99082.7715 & CSP30 & Yes & 126 & 11.62 & 0 & 11.48 & 16.86 & 9.86 & 7.35 \\
\hline Cluster-111962.0 & CSP31 & Yes & 121 & 18.58 & 0 & 18.48 & 24.69 & 16 & 14.29 \\
\hline Cluster-99082.42417 & CSP32 & Yes & 124 & 4.21 & 0 & 2.19 & 6.22 & 3.36 & 2.47 \\
\hline Cluster-99082.28064 & CSP33 & Yes & 121 & 254.21 & 166.1 & 180.14 & 292.03 & 286.28 & 206.62 \\
\hline Cluster-68449.0 & CSP34 & Yes & 119 & 0.5 & 0 & 0.96 & 1.45 & 0.98 & 0 \\
\hline Cluster-99082.40634 & CSP35 & Yes & 120 & 61.8 & 50.51 & 43 & 62.14 & 105.6 & 48.74 \\
\hline Cluster-99082.13305 & $O B P 1$ & Yes & 145 & 241.86 & 295.68 & 449.66 & 83.24 & 141.97 & 157.73 \\
\hline Cluster-96513.0 & $O B P 2$ & Yes & 139 & 1.18 & 0 & 2.03 & 2.42 & 2.03 & 0.86 \\
\hline Cluster-20697.0 & $O B P 3$ & Yes & 142 & 0 & 0 & 0.85 & 0.37 & 2.38 & 1.34 \\
\hline Cluster-99082.1411 & $O B P 4$ & Yes & 149 & 16.3 & 17.65 & 19.47 & 19.5 & 14.33 & 19.22 \\
\hline Cluster-99082.41850 & OBP5 & Yes & 141 & 30.23 & 0 & 27.49 & 54.24 & 35.64 & 19.13 \\
\hline
\end{tabular}




\begin{tabular}{|c|c|c|c|c|c|c|c|c|c|}
\hline \multirow[t]{2}{*}{ Gene ID } & \multirow[t]{2}{*}{ Gene name } & \multirow{2}{*}{$\begin{array}{l}\text { Complete } \\
\text { ORF }\end{array}$} & \multirow{2}{*}{$\begin{array}{c}\text { ORF } \\
\text { (aa) }\end{array}$} & \multicolumn{6}{|c|}{ FPKM } \\
\hline & & & & C_1 & C_2 & C_3 & X_1 & X_2 & X_3 \\
\hline Cluster-99082.11505 & OBP6 & No & 117 & 3.25 & 13 & 14.01 & 4.94 & 5.83 & 3.58 \\
\hline Cluster-99082.11785 & $O B P 7$ & Yes & 149 & 54.03 & 53.21 & 55.26 & 54.02 & 70.76 & 43.9 \\
\hline Cluster-99082.8686 & OBP8 & No & 87 & 23.91 & 88.29 & 159.36 & 30.18 & 42.23 & 82.89 \\
\hline Cluster-75015.0 & $O B P 9$ & Yes & 150 & 4.56 & 7.28 & 6.01 & 8.27 & 13.03 & 14.95 \\
\hline Cluster-70529.0 & OBP10 & Yes & 183 & 3.4 & 0 & 2.96 & 3.9 & 2.81 & 2.16 \\
\hline Cluster-99082.17908 & $O B P 11$ & Yes & 148 & 978.02 & 866.45 & 1106.45 & 901.3 & 923.1 & 778.06 \\
\hline Cluster-80695.0 & OBP12 & No & 114 & 0.87 & 5.89 & 14.26 & 19.85 & 3.47 & 9.28 \\
\hline Cluster-71856.0 & OBP13 & Yes & 152 & 9.59 & 14.22 & 20.46 & 13.72 & 5.17 & 9.37 \\
\hline Cluster-99082.45233 & OBP14 & No & 147 & 36.31 & 42.52 & 34.54 & 147.31 & 74.82 & 61.56 \\
\hline Cluster-57019.0 & $O B P 15$ & No & 147 & 0 & 2.05 & 0 & 1.61 & 0 & 0 \\
\hline Cluster-86071.0 & OBP16 & Yes & 184 & 0.84 & 2.18 & 0.4 & 0.78 & 0 & 0 \\
\hline Cluster-99082.5240 & OBP17 & Yes & 152 & 22.76 & 0 & 16.84 & 31.49 & 23.03 & 15.26 \\
\hline Cluster-68485.0 & OBP18 & Yes & 137 & 0.81 & 0 & 3.47 & 1.15 & 1.95 & 1.12 \\
\hline Cluster-84571.0 & OBP19 & Yes & 140 & 2.1 & 4 & 9.57 & 13.12 & 9.65 & 6.33 \\
\hline Cluster-99043.0 & OBP20 & Yes & 139 & 2.24 & 0 & 0.3 & 2.06 & 0.61 & 0.43 \\
\hline Cluster-99082.30661 & $O B P 21$ & Yes & 141 & 44.4 & 0 & 40.1 & 64.65 & 42.59 & 28.28 \\
\hline Cluster-99082.8989 & $O B P 22$ & No & 115 & 0 & 0 & 0.93 & 0 & 7.93 & 4.19 \\
\hline Cluster-99082.9946 & $O B P 23$ & Yes & 141 & 6.08 & 5.41 & 0 & 2.72 & 3.12 & 2.08 \\
\hline Cluster-99082.13306 & OBP72-like* & Yes & 139 & 45.82 & 49.95 & 84.96 & 4.83 & 5.5 & 22.16 \\
\hline Cluster-99082.11504 & OBP83a-like & Yes & 141 & 101.57 & 119.57 & 247 & 40.39 & 29.77 & 81.27 \\
\hline Cluster-99082.24868 & OBP69a-like* & Yes & 137 & 632.67 & 750.5 & 926.75 & 166.58 & 341.25 & 309.07 \\
\hline Cluster-99082.38297 & OBP56d & Yes & 142 & 30.42 & 82.25 & 66.87 & 79.49 & 100.67 & 102.71 \\
\hline Cluster-99082.28069 & OBP84a & Yes & 171 & 209.03 & 157.81 & 240.33 & 94.39 & 91.75 & 175.83 \\
\hline Cluster-99082.17039 & $G O B P 1$ & Yes & 165 & 216.8 & 298.51 & 562.62 & 93.55 & 117.35 & 149.49 \\
\hline Cluster-99082.19529 & $G O B P 2$ & Yes & 164 & 1106.23 & 1590.77 & 2493.64 & 522.32 & 780.68 & 855.93 \\
\hline Cluster-99082.19683 & GOBP3 & Yes & 160 & 8.86 & 0 & 9.95 & 13.2 & 10.23 & 6.07 \\
\hline Cluster-76163.1 & $P B P 1$ & Yes & 163 & 2.74 & 0 & 0 & 0.41 & 0 & 1.16 \\
\hline Cluster-99082.32171 & $P B P 2$ & Yes & 163 & 1644.81 & 1863.84 & 1969.72 & 810.34 & 1191.06 & 933.66 \\
\hline Cluster-99082.23894 & $P B P 3$ & Yes & 169 & 555.26 & 797.63 & 1336.72 & 251.49 & 301.99 & 416.73 \\
\hline Cluster-99082.4414 & PBP4 & Yes & 163 & 8.16 & 0 & 8.49 & 10.23 & 6.99 & 4.78 \\
\hline Cluster-99082.44500 & PBP5 & Yes & 162 & 8.42 & 0 & 8.22 & 13.71 & 9.57 & 6.68 \\
\hline Cluster-99082.44837 & PBP6 & Yes & 174 & 4.69 & 0 & 3.97 & 6.15 & 3.83 & 2.8 \\
\hline Cluster-99082.14291 & ORl-like & No & 269 & 4.67 & 0.84 & 0.24 & 0.41 & 0.09 & 0.84 \\
\hline Cluster-99082.27417 & Orco & Yes & 474 & 13.08 & 10.48 & 15.86 & 1.85 & 1.23 & 3.74 \\
\hline Cluster-81186.0 & OR3 & No & 224 & 0 & 0 & 0 & 0 & 0 & 0 \\
\hline Cluster-108539.0 & OR4 & No & 417 & 2.3 & 1.28 & 2.24 & 0.59 & 0.51 & 1 \\
\hline Cluster-112101.0 & OR5 & No & 151 & 1.57 & 1.68 & 6.01 & 0 & 3.79 & 5.45 \\
\hline Cluster-99082.41259 & OR6 & No & 229 & 1.22 & 0.4 & 1.33 & 0 & 0 & 0 \\
\hline Cluster-67201.0 & OR7 & Yes & 409 & 2.62 & 2.94 & 0 & 0 & 0 & 0 \\
\hline Cluster-111607.2 & OR8 & No & 197 & 0 & 0 & 0 & 0 & 0 & 0 \\
\hline Cluster-99082.19046 & OR9 & No & 109 & 4.59 & 6.36 & 4.43 & 0 & 0 & 4.62 \\
\hline
\end{tabular}




\begin{tabular}{|c|c|c|c|c|c|c|c|c|c|}
\hline \multirow[t]{2}{*}{ Gene ID } & \multirow[t]{2}{*}{ Gene name } & \multirow{2}{*}{$\begin{array}{c}\text { Complete } \\
\text { ORF }\end{array}$} & \multirow{2}{*}{$\begin{array}{l}\text { ORF } \\
\text { (aa) }\end{array}$} & \multicolumn{6}{|c|}{ FPKM } \\
\hline & & & & C_1 & C_2 & C_3 & X_1 & $X \_2$ & X_3 \\
\hline Cluster-73375.2 & OR10 & Yes & 371 & 2.5 & 0.22 & 0.44 & 0 & 0.2 & 0.57 \\
\hline Cluster-72331.0 & OR11 & No & 237 & 1.17 & 0.38 & 2.37 & 0.27 & 2.22 & 1.71 \\
\hline Cluster-92187.0 & OR12 & Yes & 453 & 1.65 & 0.45 & 0 & 0 & 0 & 0 \\
\hline Cluster-90816.0 & OR13a-like & Yes & 434 & 0 & 0 & 0.15 & 0.01 & 0.01 & 0 \\
\hline Cluster-93549.0 & OR14 & No & 95 & 0 & 22.13 & 14.85 & 0 & 0 & 0 \\
\hline Cluster-35945.3 & OR15 & No & 190 & 0.73 & 1.94 & 1.08 & 0 & 0 & 0.13 \\
\hline Cluster-99082.17892 & OR19 & No & 177 & 5.03 & 9.75 & 0 & 1.97 & 4.41 & 0 \\
\hline Cluster-10752.0 & OR21 & No & 113 & 0 & 0 & 0 & 6.24 & 0 & 0 \\
\hline Cluster-99082.17689 & OR22 & No & 141 & 1.2 & 2.73 & 0.47 & 0 & 0 & 0 \\
\hline Cluster-95812.0 & OR25 & No & 66 & 3.81 & 0 & 4.19 & 0 & 0 & 0 \\
\hline Cluster-99082.20050 & OR26 & No & 389 & 3.19 & 3.18 & 4.65 & 0 & 1.43 & 0 \\
\hline Cluster-99082.9723 & OR29 & Yes & 394 & 12.53 & 8.75 & 13.72 & 0.94 & 1.21 & 1.54 \\
\hline Cluster-99082.47097 & OR30a-like & No & 296 & 4.52 & 2.29 & 1.76 & 0 & 1.06 & 0 \\
\hline Cluster-99082.39240 & OR33a-like & No & 418 & 6.41 & 14.66 & 7.51 & 2.9 & 3.7 & 4.69 \\
\hline Cluster-20109.0 & OR38 & No & 112 & 0 & 0 & 11.42 & 0 & 0 & 0 \\
\hline Cluster-89415.0 & OR42a-like & No & 183 & 2.57 & 0 & 2.64 & 0 & 0 & 2.86 \\
\hline Cluster-31917.0 & OR46a & No & 292 & 0.48 & 0.83 & 2.48 & 0 & 0.36 & 0.52 \\
\hline Cluster-86629.0 & OR67c-like & Yes & 453 & 1.68 & 1.61 & 1.55 & 0.21 & 0.26 & 0.41 \\
\hline Cluster-68248.0 & OR85b-like & No & 237 & 0.74 & 0 & 0 & 0 & 0 & 0.26 \\
\hline Cluster-29101.0 & OR85c-like & No & 181 & 0 & 2.56 & 2.61 & 0 & 0 & 0 \\
\hline Cluster-91085.0 & OR85e & No & 122 & 3.44 & 0 & 0 & 0 & 0 & 0 \\
\hline Cluster-90531.0 & OR92a & No & 232 & 1.06 & 0.39 & 0.87 & 0 & 0 & 0 \\
\hline Cluster-14757.3 & OR94a-like & Yes & 395 & 1.81 & 0.6 & 2.87 & 1.43 & 0 & 2.08 \\
\hline Cluster-82570.0 & OR94b-like & No & 212 & 2.49 & 0.46 & 3.55 & 0 & 0 & 0 \\
\hline Cluster-99082.45453 & $I R 1$ & No & 175 & 0 & 0 & 0 & 0 & 0 & 0 \\
\hline Cluster-99082.27319 & $I R 8 a$ & Yes & 729 & 1.17 & 2.22 & 1.92 & 0.55 & 0.31 & 1.19 \\
\hline Cluster-97999.0 & $\operatorname{IR} 21 a$ & Yes & 624 & 2.1 & 1.84 & 1.46 & 2.17 & 0.95 & 1.57 \\
\hline Cluster-99082.21385 & $I R 25 a$ & Yes & 931 & 3.68 & 2.35 & 2.77 & 0.9 & 0 & 2.62 \\
\hline Cluster-86747.1 & IR4la & No & 692 & 5.12 & 3.08 & 3.17 & 4.38 & 0.66 & 2.58 \\
\hline Cluster-69139.0 & IR60a & No & 202 & 1.18 & 0 & 2.07 & 0 & 0 & 1.07 \\
\hline Cluster-99082.43547 & IR64a & Yes & 603 & 4.67 & 2.99 & 2.98 & 2.2 & 2.65 & 5.87 \\
\hline Cluster-101108.0 & IR68a & No & 182 & 0 & 2.11 & 0 & 1.14 & 0 & 0 \\
\hline Cluster-110656.0 & $I R 75 a$ & No & 279 & 5.51 & 0 & 0 & 0 & 3.76 & 7.5 \\
\hline Cluster-88706.0 & $I R 75 d$ & No & 82 & 0 & 0 & 0 & 0 & 0 & 0 \\
\hline Cluster-80057.0 & $I R 75 P$ & No & 114 & 0 & 0 & 11.2 & 0 & 0 & 0 \\
\hline Cluster-40640.1 & $I R 75 q$ & No & 633 & 0.73 & 2.12 & 1.92 & 0.16 & 0 & 0 \\
\hline Cluster-99082.46332 & $I R 76 b$ & Yes & 547 & 3.44 & 3.62 & 4.41 & 0.76 & 1.07 & 1.13 \\
\hline Cluster-95085.0 & $\operatorname{IR} 87 a$ & No & 235 & 0.75 & 1.55 & 1.28 & 0.69 & 1.42 & 0 \\
\hline Cluster-99082.29255 & SNMP1 & Yes & 525 & 10.92 & 6.71 & 12.31 & 1.97 & 1.78 & 3.22 \\
\hline Cluster-99082.27417 & SNMP2 & Yes & 521 & 61.48 & 75.64 & 116.44 & 47.51 & 44.88 & 72.4 \\
\hline
\end{tabular}




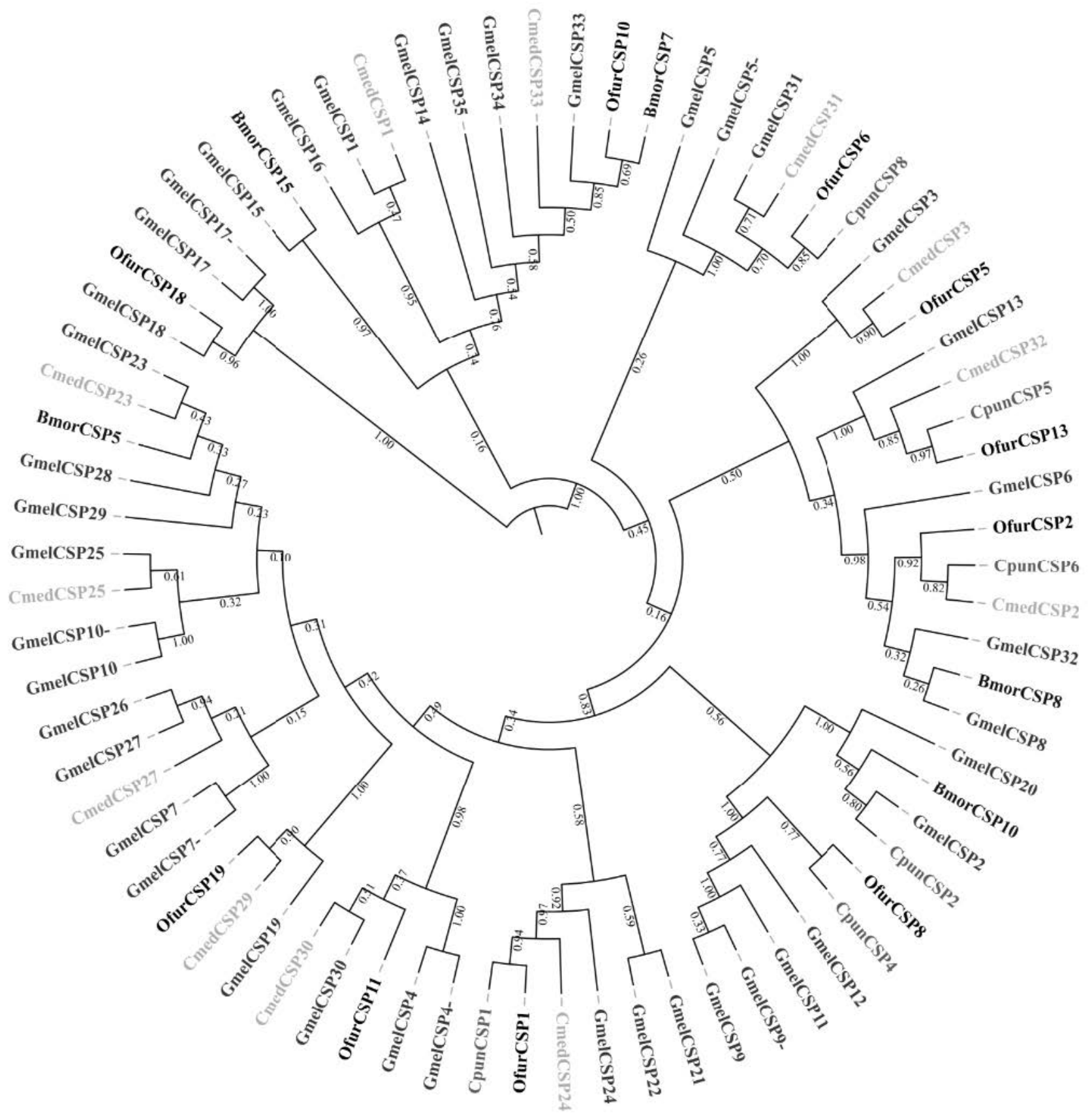

Fig. 2. Phylogenetic tree of candidate GmelCSPs with known lepidopteran chemosensory protein (CSP) sequences. Gmel, Galleria mellonella; Csup, Chilo suppressalis; Ofur, Ostrinia furnacalis; Cmed, Cnaphalocrocis medinalis; Cpun, Conogethes punctiferalis; Bmor, Bombyx mori.

\section{Chemosensory proteins}

A total of 35 unigenes were annotated to putative CSPs, all of which were predicted to have four cysteines, and they consisted of a full-length ORF encoding 97-147 amino acids. The neighbor-joining tree analysis revealed that all 35 sequences were clustered with at least 1 lepidopteran orthologous gene, and the CSPs were clearly observed (Fig. 2). The unigenes corresponding to CSPs were designated according to the obtained CSP data.

Olfactory receptors

A total of 33 ORs were identified from the assembled 
unigenes, which belonged to the seven-transmembrane receptor superfamily. Among them, eight ORs (Orco, OR7, OR10, OR12, OR13a-like, OR29, OR67c-like and OR94alike) contained full-length ORFs with 5-7 transmembrane domains. As could be seen from the phylogenetic tree in
Figure 3, the $O R 2$ sequence was $99 \%$ identical to $C p u n O R 2$ and CmedOrco, and thus we labeled it as GmelOrco, while other ORs were classified into distinct clades with known ORs.

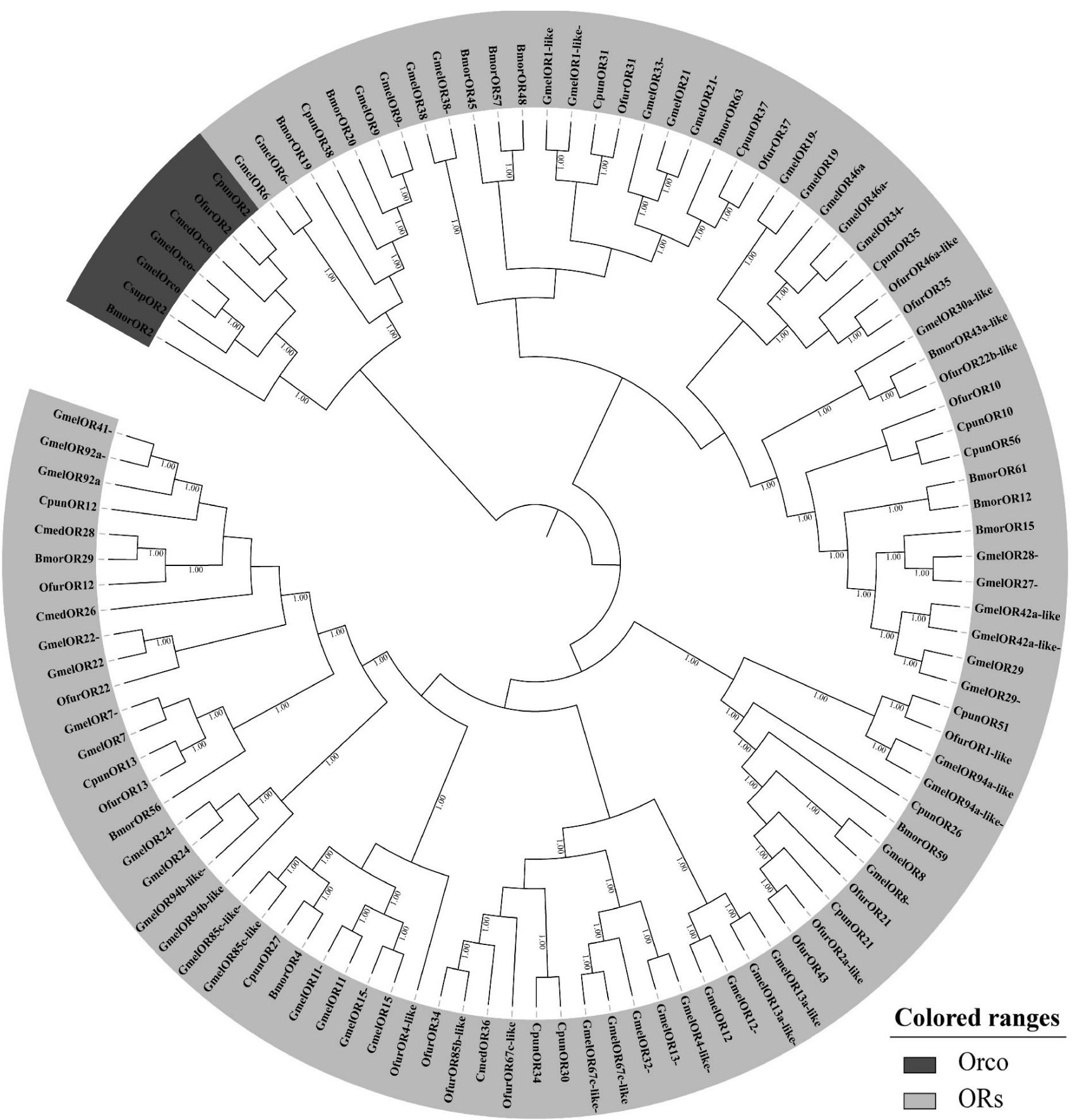

Fig. 3. Phylogenetic tree of candidate GmelORs with known lepidopteran olfactory receptor (OR) sequences. Gmel, Galleria mellonella; Csup, Chilo suppressalis; Ofur, Ostrinia furnacalis; Cmed, Cnaphalocrocis medinalis; Cpun, Conogethes punctiferalis; Bmor, Bombyx mori. The clade in purple indicates the Orco receptor gene clade. 


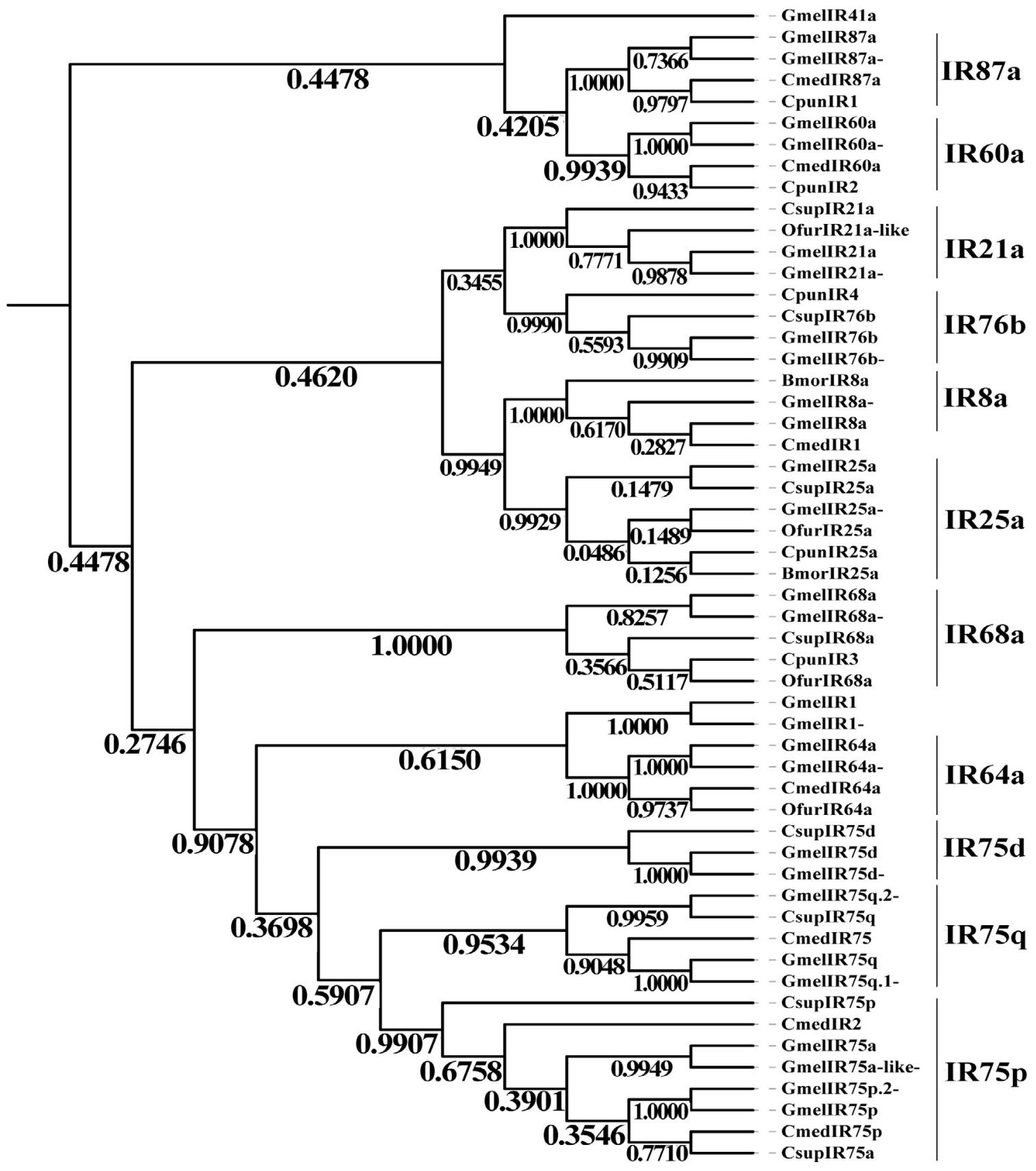

Fig. 4. Phylogenetic tree of candidate GmelIRs with known lepidopteran ionotropic receptor (IR) sequences. Gmel, Galleria mellonella; Csup, Chilo suppressalis; Ofur, Ostrinia furnacalis; Cmed, Cnaphalocrocis medinalis; Bmor, Bombyx mori; Cpun, Conogethes punctiferalis.

\section{Ionotropic receptors}

We identified 14 transcripts encoding candidate IRs, including IR $8 a$ and IR25a (members of highly conserved IR co-receptors). Among these, 5 IRs (IR8a, IR21a, IR25a, $I R 64 a$ and $I R 76 b)$ contained full-length ORFs encoding a protein of 547 amino acids. As shown in Figure 4, most
IRs were clustered together with their orthologs into a distinct clade.

Sensory neuron membrane proteins

SNMP transcripts with two TMDs were identified in the transcriptome of G. mellonella, including SNMP1 and 
SNMP2. Moreover, intact ORFs with 525 and 521 amino acids were observed for $S N M P 1$ and $S N M P 2$, respectively. Figure 5 shows the phylogenetic tree constructed according to 31 SNMP sequences from 19 species. Notably, the insect SNMPs were assigned to two highly conserved, distinguishable classes, namely, SNMP1 and SNMP2.

\section{Expression profiles of the candidate chemosensory genes}

The expression levels of the 121 chemosensory unigenes in six cDNA libraries were determined with the FPKM index. The differential expression profiles revealed that several chemosensory genes (CSP1, CSP 2, CSP3, CSP4, CSP6, CSP7, CSP10, CSP28, CSP33,
OBP1, OBP11, OBP69a-like, OBP84a, GOBP1, GOBP2, $P B P 2$ and $P B P 3$ ) were overexpressed in female and male antennae (FPKM > 100), and CSP6 exhibited the highest expression $(\mathrm{FPKM}=31189.81)$. Moreover, some genes were specifically detected in the sexual state, although their expression was extremely low. For example, CSP8, CSP25, CSP29, OR6, OR7, OR12, OR38, OR85c-like, OR85e, OR92a, OR94b-like and IR75p were expressed exclusively in the female antennae, while $O R 21$ was detected only in the male antennae. Furthermore, OR, IR and SNMP genes exhibited relatively low expression in each sample (FPKM $<100$ ), and the two SNMPs demonstrated higher FPKM values in the female antennae than in the male antennae.

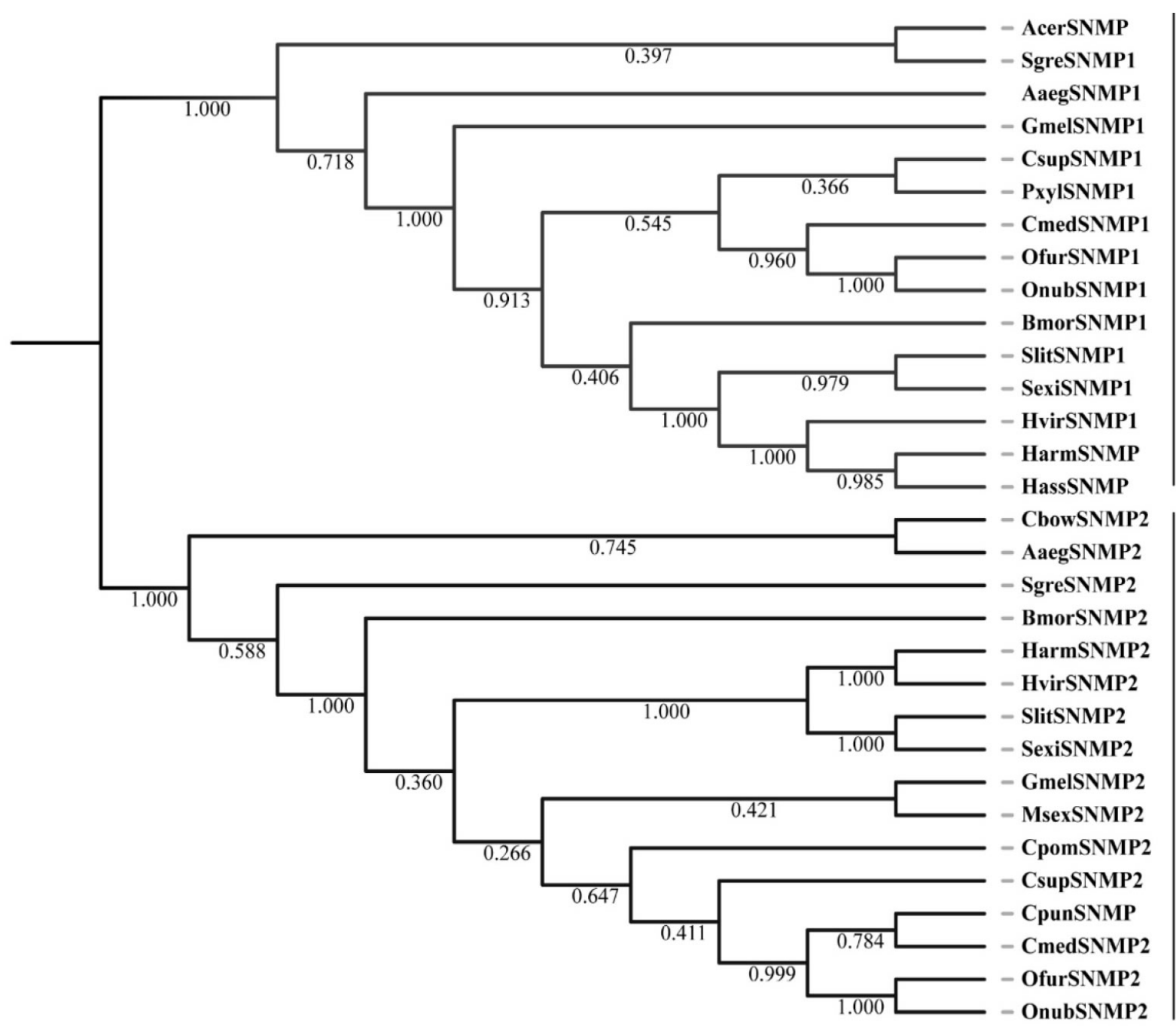

SNMP1

SNMP2

Fig. 5. Phylogenetic tree of candidate GmelSNMPs with known sensory neuron membrane protein (SNMP) sequences. Aaeg, Aedes aegypti; Acer, Apis cerana; Bmor, Bombyx mori; Cbow, Colaphellus bowringi; Cmed, Cnaphalocrocis medinalis; Cpom, Cydia pomonella; Cpun, Conogethes punctiferalis; Csup, Chilo suppressalis; Gmel, Galleria mellonella; Harm, Helicoverpa armigera; Hass, Helicoverpa assulta; Hvir, Heliothis virescens; Msex, Manduca sexta; Ofur, Ostrinia furnacalis; Onub, Ostrinia nubilalis; Pxyl, Plutella xylostella; Sexi, Spodoptera exigua; Sgre, Schistocerca gregaria; Slit, Spodoptera litura. 
A

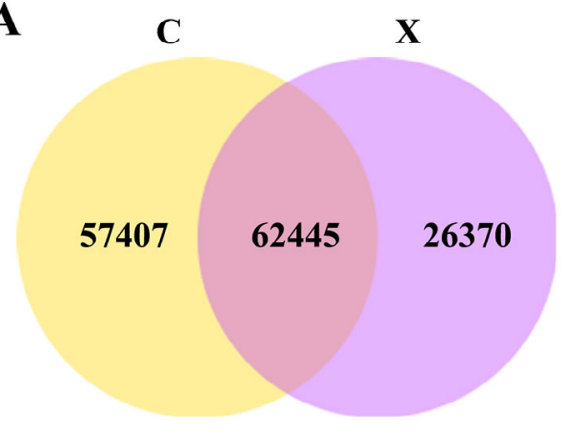

B

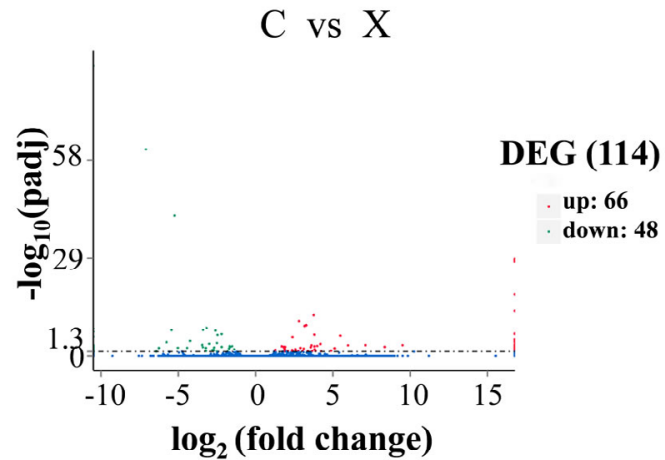

C

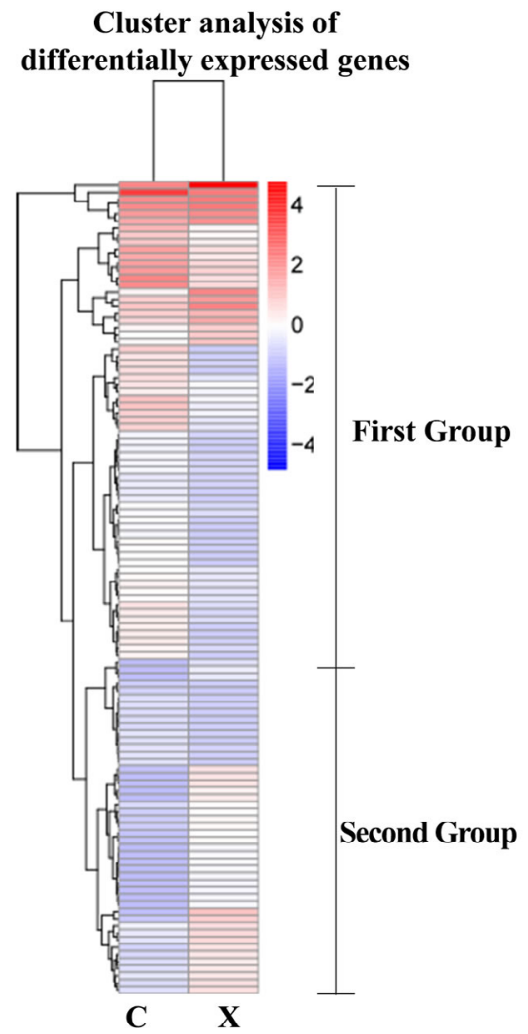

Fig. 6. Bioinformatic analyses of differentially expressed genes (DEGs). A, Venn diagrams showing the number of genes expressed in the two groups; B, DEG distribution between the two treatment groups (C, Female; X, Male); $\mathbf{C}$, cluster analysis of DEGs.

\section{Analysis of DEGS}

Next, DEG analysis was carried out, and the Venn diagram in Figure 6A shows the overlap of 62,445 genes between the two groups. Notably, 57,407 genes were expressed exclusively in the female antennae, while 26,370 were found only in the male antennae. Moreover, 114 DEGs were identified, of which 5 were chemosensory-related genes such as 2 CSPs (CSP7 and CSP10), 2 OBPs (OBP72-like and OBP69a-like) and $1 \mathrm{OR}$ (OR29). Among these DEGs, 66 were upregulated and 48 were downregulated in the female antennae (Fig. 6B). The cluster analysis revealed that the DEGs between females (C) and males (X) were assigned to two main groups: (i) gene upregulation and (ii) gene downregulation (Fig. 6C).

The GO enrichment analysis of DEGs was performed using the GOseq method with Wallenius non-central hypergeometric distribution (Young et al., 2012). The DEGs were mostly enriched in the "binding" category, followed by "metabolic process", "cellular process" and "single-organism process" categories (Fig. 7). More olfactory-related GO terms were related to the upregulated DEGs compared with the downregulated DEGs. These olfactory-related GO terms were associated with the molecular function (binding, catalytic activity, protein binding and ion binding), biological process (metabolic process, cellular process and single-organism process), and cellular component (cell and cell part).

In addition, our results demonstrated that all DEGs were mapped to 28 reference KEGG pathways. These DEGs were remarkably enriched in "ubiquitin mediated proteolysis", "signaling pathways regulating pluripotency of stem cells", and "renal cell carcinoma and pathways in cancer" (Fig. 8). Besides, several enriched pathways were associated with cAMP, hypoxia-inducible factor- 1 (HIF1), Hippo, mitogen-activated protein kinase (MAPK), T cell receptor, and glucagon signaling pathways.

\section{Gene expression level validation by $q R T-P C R$}

To verify the results of the transcriptome analysis, we carried out qRT-PCR to detect the expression of 16 DEGs (2 CSPs, 2 OBPs, 1 OR, 1 gustatory receptor, 2 P450s, 1 juvenile hormone binding protein and 7 other genes). The results demonstrated that the expression patterns of 15 out of the 16 selected genes were consistent with those generated from the RNA-Seq analysis (Table V), thus implying the reliability of the sequencing data. 


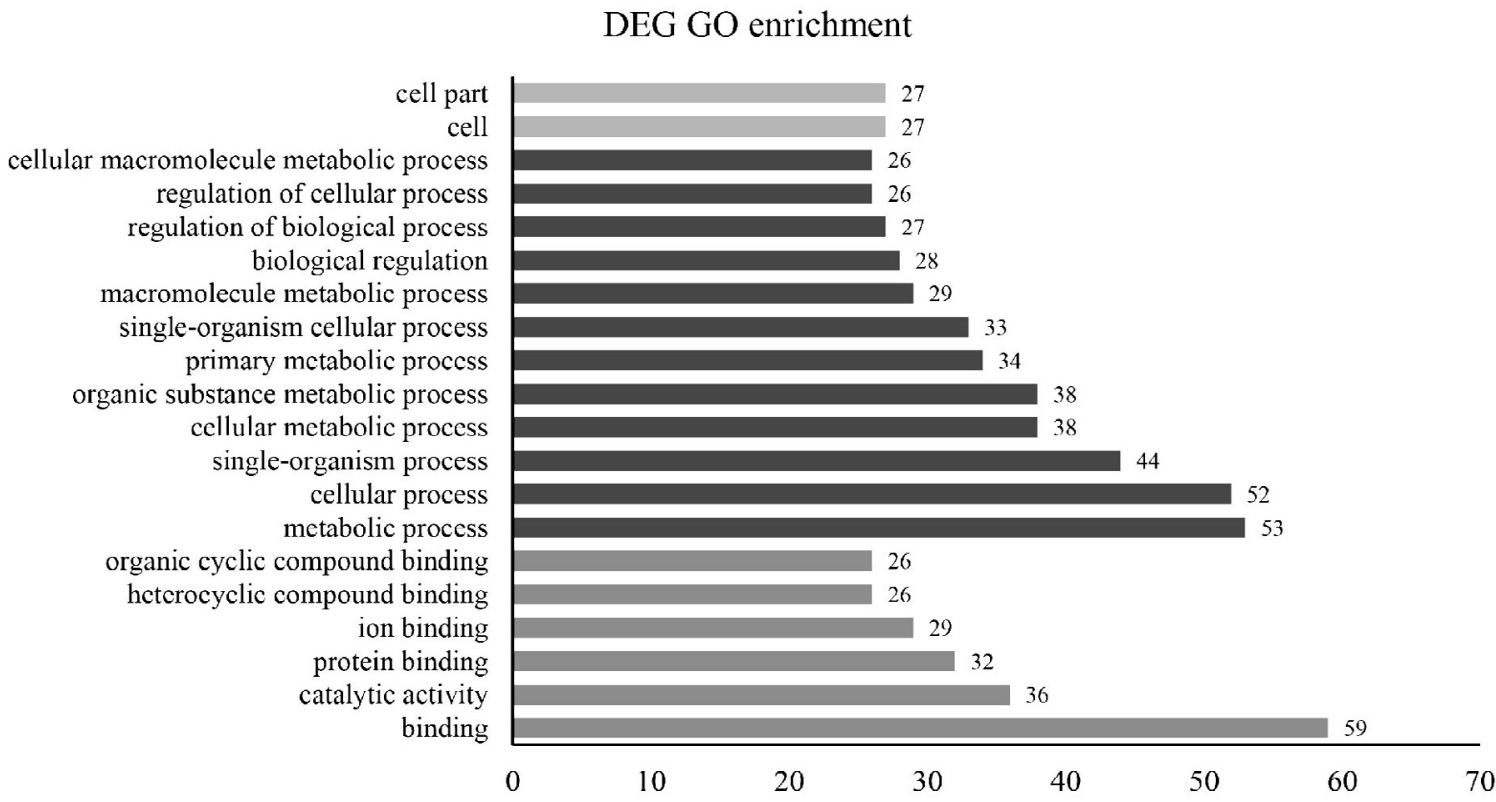

Fig. 7. Bar graph showing enriched gene ontology (GO) terms for differentially expressed genes (DEGs) between the female and male antennae.

Statistics of Pathway Enrichment

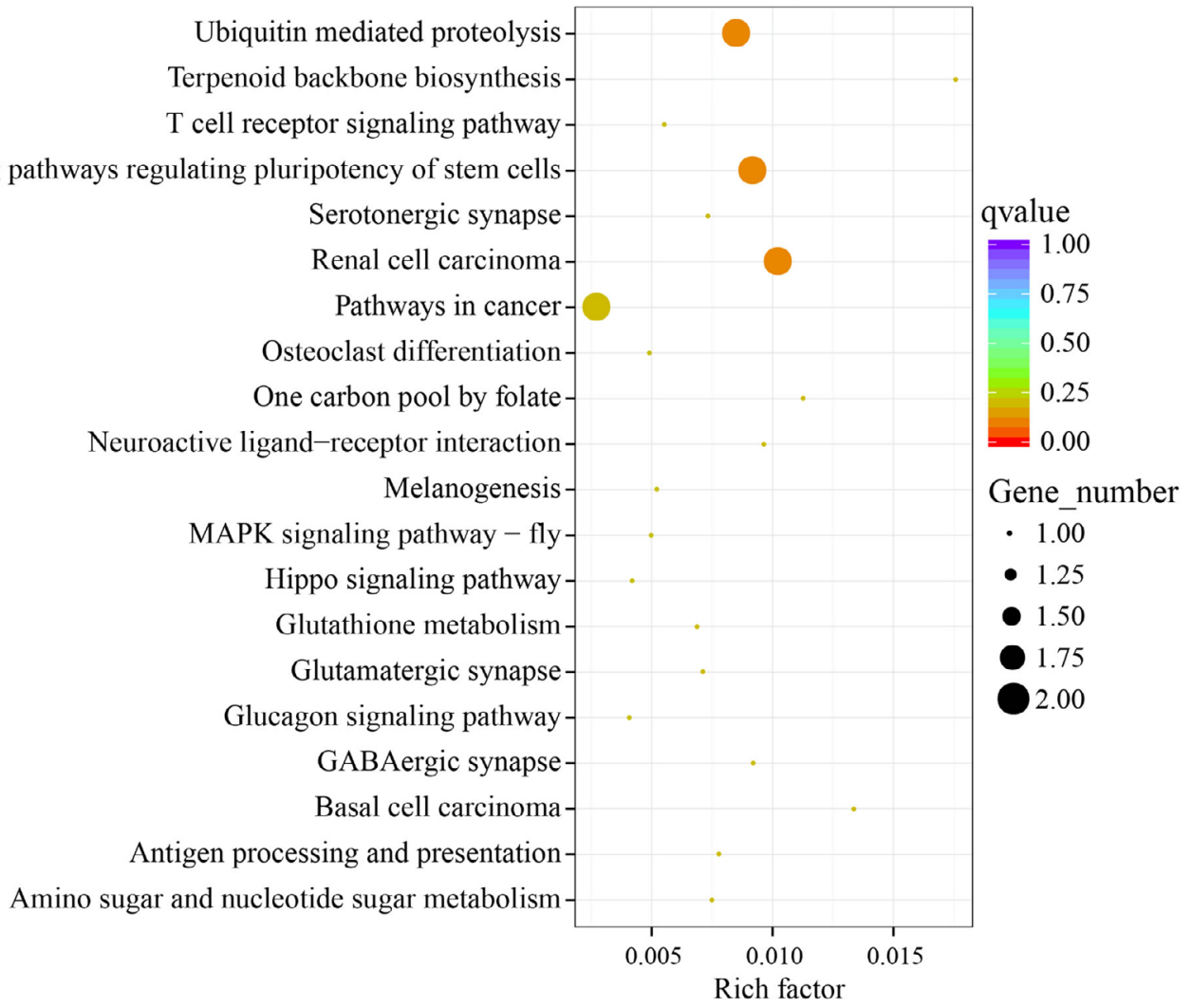

Neuroactive ligand-receptor interaction- .

Fig. 8. Differentially expressed genes (DEG)-enriched Kyoto Encyclopedia of Genes and Genomes (KEGG) pathway. 
Table V. RNA-Seq and qRT-PCR analyses of DEGs between male and female $G$. mellonella samples.

\begin{tabular}{lll}
\hline \multirow{2}{*}{ Gene name } & \multicolumn{2}{c}{$\log _{2}$ (fold change) } \\
\cline { 2 - 3 } & RNA-Seq & qRT-PCR \\
\hline CSP 3 & -1.49 & -1.23 \\
CSP 34 & -1.30 & -1.94 \\
OBP 8 & 2.47 & 1.60 \\
OBP 17 & 1.49 & 0.79 \\
OR 15 & 3,24 & 2.43 \\
cytochrome P450-1 & 2.97 & 2.73 \\
cytochrome P450-2 & 2.02 & 1.23 \\
gustatory receptor 67 & -3.32 & -6.37 \\
juvenile hormone binding protein & -6.87 & -4.41 \\
3-hydroxyacyl-CoA & -2.25 & -2.31 \\
S9-desaturase & -5.01 & -4.91 \\
allantoicase & -2.80 & -3.42 \\
trypsin & -2.52 & -3.44 \\
troponin & -2.56 & -1.75 \\
cuticular protein & -6.34 & -4.78 \\
blastopia polyprotein & 6.65 & 0.61 \\
\hline
\end{tabular}

\section{DISCUSSION}

Pollinators provide an important ecosystem service by enhancing the yields of wild and crop plants globally ( $\mathrm{Li}$ et al., 2015). Increasing evidence has shown the declines in both wild and domesticated insect pollinators (Kwadha et al., 2017). G. mellonella is considered a key factor for the decreases in both native and feral honey bee colonies, especially in tropical and sub-tropical regions (Kwadha et al., 2017). Olfaction is an important attribute of smell for the greater wax moths to reproduce and survive (Zhao et al., 2019). In the present work, the antennal transcriptomes of both sexes of the greater wax moths were sequenced and analyzed. We identified 121 chemosensory gene transcripts (37 OBPs, 35 CSPs, 33 ORs, 14 IRs and 2 SNMPs), and which might provide base data to elucidate the olfactory recognition mechanism of $G$. mellonella. Numerous candidate chemosensory genes identified in this study are comparable with those reported in a recent study (Zhao et al., 2019), with 22 OBPs, 20 CSPs, 46 ORs, 25 IRs and 2 SNMPs. The reduced number of ORs and IRs may be attributed to different sampling time and $G$. mellonella life-cycle, as the greater wax moth are more active during the first three days and their activities reduce with an increase in age (Li et al., 2019).

Courtship behavior is essential for animal reproduction (Grosjean et al., 2011; Jiang et al., 2016). To mate, animals have evolved a wide variety of pheromone release and detection patterns (Zhang et al., 2015c). G. mellonella is an interesting insect to study because males attract females (Kwadha et al., 2017). Pheromone- binding proteins (PBPs) are a subgroup of OBPs, which play a crucial role in regulating olfactory process (Liu et al., 2012). In this research, we identified six transcripts (PBP1, PBP2, PBP3, PBP4, PBP5 and PBPO) encoding candidate PBP genes based on their similarity with the PBPs from other lepidopterans and the physiological analysis. The number of PBP genes identified was slightly higher than that reported in other lepidopteran insects, and these six sequences were detected in the antennae of both female and male wax moths. Thus, we speculate that the PBPs in G. mellonella are different from those in other lepidopteran species, as the expression of PBPs is malebiased and antenna-predominant in most lepidopterans during the synthesis of male pheromones (Zhao et al., 2019). However, the function of PBPs in G. mellonella remains to be fully elucidated.

SNMPs were first discovered in pheromoneresponsive neurons, which could influence the detection of pheromones (Rogers et al., 2001). At present, the molecular mechanism underlying the functions of insect SNMPs is poorly understood (Li et al., 2015). In this study, we successfully identified two SNMPs (SNMP1 and $S N M P 2)$ that were not differentially expressed between the female and male antennae, although a higher FPKM value was observed in the female antennae than in the male antennae. These findings are consistent with those reported in other known lepidopterans, suggesting that the SNMPs in G. mellonella may play a similar role as in $D$. melanogaster and other moths.

To assess the differential expression patterns of chemosensory-related genes in both female and male antennae, RNA-Seq was performed to compare the levels of DEGs. The results indicated that the number of downregulated DEGs (66) was slightly lower than that of upregulated DEGs (48) between the two samples. Among these DEGs, we identified three (OBP72-like, OBP69alike and OR29) and two (CSP7 and CSP10) genes with remarkably higher expression levels in the female and male antennae, respectively. According to the functions of insect OBPs, CSPs and ORs (Li et al., 2015), the femalebiased OBP72-like, OBP69a-like and OR29 genes are responsible for the detection of sex pheromones released by males or odors critical to female-specific behaviors (e.g., searching bee-comb hosts for oviposition), while male-biased CSP7 and CSP10 genes can detect odors critical to male-specific behaviors. Nevertheless, the sexspecific functions of these chemosensory-related DEGs should be further investigated.

GO analysis demonstrated that the 114 DEGs were most significantly enriched in molecular function (e.g., binding). These included protein binding, ion binding, heterocyclic compounding, and organic cyclic compound binding. Considering that the antennae are 
critical olfactory appendages in an olfactory system that interact with different types of chemical stimuli (Zhao et al., 2019), it is reasonable that the identified DEGs are involved in binding. The KEGG analysis revealed that signal transduction pathways (e.g., stem cells, $\mathrm{T}$ cell receptor, MAPK, glucagon, calcium, HIF-1, cAMP and Hippo signaling pathways) were found to be markedly enriched and closely associated with the antenna (as the primary organ of odor binding and signal transduction).

\section{CONCLUSION}

The analysis of antennal transcriptomic data revealed 121 putative chemosensory genes in G. mellonella and 114 DEGs between the male and female antennae. Furthermore, our method successfully detected chemosensory genes with very low expression, which could provide essential information to further investigate the underlying olfactory recognition mechanism in G. mellonella and serve as a platform for further functional analyses of the related genes. This may ultimately lead to the identification of a suitable male pheromone that can be used as a bait for trapping G. mellonella. Such pest management strategy can treat and/or prevent moth infestations of honey bees and, consequently, improve the health of honey bee colonies worldwide.

\section{ACKNOWLEDGEMENTS}

This work was supported by the National Natural Science Foundation of China (31640080) and China Agriculture Research System (CARS-44-SYZ 16). We acknowledge the help of Xinyu Li, Wenting Su, Yujia Feng, and Denglong Long with the experiments. We greatly appreciate the assistance of the scientists of Novogene Bioinformatics Technology Co., Ltd. in sequencing the DEG libraries. We also thank EditSprings for providing language editing services.

\section{Statement of conflicts of interest}

We declare that we do not have any commercial or associative interest that represents a conflict of interest in connection with the work submitted.

\section{REFERENCES}

Benton, R., Vannice, K.S., Gomez-Diaz, C. and Vosshall, L.B., 2009. Variant ionotropic glutamate receptors as chemosensory receptors in Drosophila. Cell, 136: 149-162. https://doi.org/10.1016/j. cell.2008.12.001

Bombelli, P., Howe, C.J. and Bertocchini, F., 2017. Polyethylene bio-degradation by caterpillars of the wax moth Galleria mellonella. Curr. Biol., 27: R292-R293. https://doi.org/10.1016/j. cub.2017.02.060

Brand, P. and Ramírez, S.R., 2017. The evolutionary dynamics of the odorant receptor gene family in corbiculate bees. Genome Biol. Evol., 9: 20232036. https://doi.org/10.1093/gbe/evx149

Cao, D., Liu, Y., Wei, J., Liao, X., Walker, W.B., Li, J. and Wang, G., 2014. Identification of candidate olfactory genes in Chilo suppressalis by antennal transcriptome analysis. Int. J. biol. Sci., 10: 846860. https://doi.org/10.7150/ijbs.9297

Chang, X.Q., Nie, X.P., Zhang, Z., Zeng, F.F., Lv, L., Zhang, S. and Wang, M.Q., 2017. De novo analysis of the oriental armyworm Mythimna separata antennal transcriptome and expression patterns of odorant-binding proteins. Comp. Biochem. Physiol. Part D: Genomics Proteomics, 22: 120-130. https://doi.org/10.1016/j.cbd.2017.03.001

Charriere, J.D., and Imdorf, A., 1999. Protection of honey combs from wax moth damage. Am. Bee J., 139: 627-630.

Cui, H.H., Gu, S.H., Zhu, X.Q., Wei, Y., Liu, H.W., Khalid, H.D., Guo, Y.Y. and Zhang, Y.J., 2017. Odorant-binding and chemosensory proteins identified in the antennal transcriptome of Adelphocoris suturalis Jakovlev. Comp. Biochem. Physiol. Part D: Genomics Proteomics., 24: 139145. https://doi.org/10.1016/j.cbd.2016.03.001

Dweck, H.K.M., Svensson, G.P., Gündüz, E.A. and Anderbrant, O., 2010. Kairomonal response of the parasitoid, Bracon hebetor Say, to the maleproduced sex pheromone of its host, the greater waxmoth, Galleria mellonella (L.). J. chem. Ecol., 36: 171-178. https://doi.org/10.1007/s10886-0109746-X

Ellis, J.D., Graham, J.R. and Mortensen, A., 2013. Standard methods for wax moth research. $J$. Apic. Res., 52: 1-17. https://doi.org/10.3896/ IBRA.1.52.1.10

Fang, S.M., Hu, B.L., Zhou, Q.Z., Yu, Q.Y. and Zhang, Z., 2015. Comparative analysis of the silk gland transcriptomes between the domestic and wild silkworms. BMC Genomics, 16: 60. https://doi. org/10.1186/s12864-015-1287-9

Grabherr, M.G., Haas, B.J., Yassour, M., Levin, J.Z., Thompson, D.A., Amit, I., Adiconis, X., Fan, L., Raychowdhury, R., Zeng, Q., Chen, Z., Mauceli, E., Hacohen, N., Gnirke, A., Rhind, N. di Palma, F., Birren, B.W., Nusbaum, C., Lindblad-Toh, K., Friedman, N. and Regev, A., 2011. Full-length transcriptome assembly from RNA-Seq data without a reference genome. Nat. Biotechnol., 29: 
644-652. https://doi.org/10.1038/nbt.1883

Grosjean, Y., Rytz, R., Farine, J.P., Abuin, L., Cortot, J., Jefferis, G.S.X.E. and Benton, R., 2011. An olfactory receptor for food-derived odours promotes male courtship in Drosophila. Nature, 478: 236-240. https://doi.org/10.1038/nature10428

Gu, S.H., Wang, S.Y., Zhang, X.Y., Ji, P., Liu, J.T., Wang, G.R., Wu, K.M., Guo, Y.Y., Zhou, J.J. and Zhang, Y.J., 2012. Functional characterizations of chemosensory proteins of the alfalfa plant bug Adelphocoris lineolatus indicate their involvement in host recognition. PLoS One, 7: e42871. https:// doi.org/10.1371/journal.pone.0042871

Han, J., Lee, C.S., Yun, C.Y., Lee, B.H., Ko, Y.G., Kang, C.S., Lee, S.D., Hwang, J.S., Kang, S.W. and Kim, H.R., 2003. Cloning and expression of male-specific protein (MSP) from the hemolymph of greater wax moth, Galleria mellonella L. Arch. Insect Biochem. Physiol., 54: 110-120. https://doi. org/10.1002/arch.10106

He, M., Zhang, Y.N. and He, P., 2015. Molecular characterization and differential expression of an olfactory receptor gene family in the whitebacked planthopper Sogatella furcifera based on transcriptome analysis. PLoS One, 10: e0140605. https://doi.org/10.1371/journal.pone.0140605

He, P., Durand, N. and Dong, S.L., 2019. Editorial: Insect olfactory proteins (from gene identification to functional characterization). Front. Physiol., 10: 01313. https://doi.org/10.3389/fphys.2019.01313

Hood, W.M., Horton, P.M. and McCreadie, J.W., 2003. Field evaluation of the red imported fire ant (Hymenoptera: Formicidae) for the control of wax moths (Lepidoptera: Pyralidae) in stored honey bee comb. J. Agric. Urban Ent., 20: 93-103.

Jiang, X., Pregitzer, P., Grosse-Wilde, E., Breer, H. and Krieger, J., 2016. Identification and characterization of two "sensory neuron membrane proteins" (SNMPs) of the desert locust, Schistocerca gregaria (Orthoptera: Acrididae). J. Insect Sci., 16: 33. https://doi.org/10.1093/jisesa/iew015

Jin, S., Zhou, X., Gu, F., Zhong, G. and Yi, X., 2017. Olfactory plasticity: Variation in the expression of chemosensory receptors in Bactrocera dorsalis in different physiological states. Front. Physiol., 8: 672.

Krams, I., Kecko, S., Kangassalo, K., Moore, F.R., Jankevics, E., Inashkina, I., Krama, T., Lietuvietis, V., Meija, L. and Rantala, M.J., 2015. Effects of food quality on trade-offs among growth, immunity and survival in the greater wax moth Galleria mellonella. Insect Sci., 22: 431-439. https://doi. org/10.1111/1744-7917.12132
Kwadha, C.A., Ong'amo, G.O., Ndegwa, P.N., Raina, S.K. and Fombong, A.T., 2017. The biology and control of the greater wax moth, Galleria mellonella. Insects, 8: 61. https://doi.org/10.3390/ insects 8020061

Li, B. and Dewey, C.N., 2011. RSEM: accurate transcript quantification from RNA-Seq data with or without a reference genome. BMC Bioinf., 12: 323. https://doi.org/10.1186/1471-2105-12-323

Liu, S., Wang, W.L., Zhang, Y.X., Zhang, B.X., Rao, X.J., Liu, X.M., Wang, D.M. and Li, S.G., 2017. Transcriptome sequencing reveals abundant olfactory genes in the antennae of the rice leaffolder, Cnaphalocrocis medinalis (Lepidoptera: Pyralidae). Entomol. Sci., 20: 177-188. https://doi. org/10.1111/ens.12253

Li, X.M., Zhu, X.Y., Wang, Z.Q., Wang, Y., He, P., Chen, G., Sun, L., Deng, D.G. and Zhang, Y.N., 2015. Candidate chemosensory genes identified in Colaphellus bowringi by antennal transcriptome analysis. BMC Genomics, 16: 1028. https://doi. org/10.1186/s12864-015-2236-3

Li, Y., Jiang, X.C., Wang, Z.W., Zhang, J.J., Katrina, K., Shahid, M., Qu, Y.F. and Tan, K., 2019. Losing the arms race: greater wax moths sense but ignore bee alarm pheromones. Insects, 10: 1-9. https://doi. org/10.3390/insects 10030081

Liu, Y., Gu, S.H., Zhang, Y.J., Guo, Y.Y. and Wang, G.R., 2012. Candidate Olfaction Genes Identified within the Helicoverpa armigera antennal transcriptome. PLoS One, 26: e48260. https://doi.org/10.1371/ journal.pone.0048260

Liu, Y.L., Guo, H., Huang, L.Q., Pelosi, P. and Wang, C.Z., 2014. Unique function of a chemosensory protein in the proboscis of two Helicoverpa species. J. exp. Biol., 217: 1821-1826. https://doi. org/10.1242/jeb.102020

Lizana, P., Machuca, J., Larama, G., Quiroz, A., Mutis, A. and Venthur, H., 2020. Mating-based regulation and ligand binding of an odorant-binding protein support the inverse sexual communication of the greater wax moth, Galleria mellonella (Lepidoptera: Pyralidae). Insect mol. Biol., 29: 337-351. https://doi.org/10.1111/imb.12638

Mortazavi, A., Williams, B.A., McCue, K., Schaeffer, L. and Wold, B., 2008. Mapping and quantifying mammalian transcriptomes by RNA-Seq. Nat. Methods, 5: 621-628. https://doi.org/10.1038/ nmeth. 1226

Pelosi, P., Mastrogiacomo, R., Iovinella, I., Tuccori, E. and Persaud, K., 2014. Structure and biotechnological applications of odorant-binding proteins. Appl. Microbiol. Biotechnol., 98: 61-70. 
https://doi.org/10.1007/s00253-013-5383-y

Rogers, M.E., Krieger, J. and Vogt, R.G., 2001. Antennal SNMPS (sensory neuron membrane proteins) of lepidoptera define a unique family of invertebrate CD36-like proteins. J. Neurobiol., 49: 47-61. https://doi.org/10.1002/neu.1065

Sanchez-Gracia, A., Vieira, F.G. and Rozas, J., 2009. Molecular evolution of the major chemosensory gene families in insects. Heredity, 103: 208-216. https://doi.org/10.1038/hdy.2009.55

Strauss, U., Human, H., Gauthier, L., Crewe, R.M., Dietemann, V., Pirk, C.W.W., 2013. Seasonal prevalence of pathogens and parasites in the savannah honeybee (Apis mellifera scutellata). J. Inverteb. Pathol., 114: 45-52. https://doi. org/10.1016/j.jip.2013.05.003

Svensson, G.P., Gündüz, E.A., Sjöberg, N., Hedenström, E., Lassance, J.M., Wang, H.L., Löfstedt, C. and Anderbrant, O., 2014. Identification, synthesis, and behavioral activity of 5,11-dimethylpentacosane, a novel sex pheromone component of the greater wax moth, Galleria Mellonella (L.). J. chem. Ecol., 40: 387-395. https://doi.org/10.1007/s10886-0140410-8

Tamura, K., Peterson, D., Peterson, N., Stecher, G., Nei, M. and Kumar, S., 2011. MEGA5: molecular evolutionary genetics analysis using maximum likelihood, evolutionary distance, and maximum parsimony methods. Mol. Biol. Evol., 28: 27312739. https://doi.org/10.1093/molbev/msr121

Traiyasut, P., Mookhploy, W., Kimura, K., Yoshiyama, M., Khongphinitbunjong, K. and Chantawannakul, P., 2016. First detection of honey bee viruses in wax moth. Chiang Mai J. Sci., 43: 695-698.

Trapnell, C., Williams, B.A., Pertea, G., Mortazavi, A., Kwan, G., van Baren, M.J., Salzberg, S.L., Wold, B.J. and Pachter, L., 2010. Transcripts assembly and quantification by RNA-Seq reveals unannotated transcripts and isoform switching during cell differentiation. Nat. Biotechnol., 28: 511-515. https://doi.org/10.1038/nbt.1621

Vieira, F.G. and Rozas, J., 2011. Comparative genomics of the odorant-binding and chemosensory protein gene families across the Arthropoda: origin and evolutionary history of the chemosensory system. Genome Biol. Evol., 3: 476-490. https://doi. org/10.1093/gbe/evr033

Vogt, R.G. and Riddiford, L.M., 1981. Pheromone binding and inactivation by moth antennae. Nature, 293: 161-163. https://doi.org/10.1038/293161a0

Wang, L., Feng, Z., Wang, X. and Zhang, X., 2010. DEGseq: an $\mathrm{R}$ package for identifying differentially expressed genes from RNA-seq data. Bioinformatics, 26: 136-138. https://doi. org/10.1093/bioinformatics/btp612

Wei, H.S., Li, K.B., Zhang, S., Cao, Y.Z. and Yin, J., 2017. Identification of candidate chemosensory genes by transcriptome analysis in Loxostege sticticalis Linnaeus. PLoS One, 12: e0174036. https://doi.org/10.1371/journal.pone.0174036

Young, M.D., Wakefield, M.J., Smyth, G.K. and Oshlack, A., 2012. Gene ontology analysis for RNA-seq: accounting for selection bias. Genome Biol., 11: R14. https://doi.org/10.1186/gb-2010-112-r14

Zhang, J., Walker, W.B. and Wang, G., 2015a. Pheromone reception in moths: from molecules to behaviors. In: Progress in molecular biology and translational science (ed. R. Glatz), Vol. 130. Academic Press, pp. 109-128. https://doi. org/10.1016/bs.pmbts.2014.11.005

Zhang, T., Coates, B.S., Ge, X., Bai, S., He, K. and Wang, Z., 2015b. Male- and female-biased gene expression of olfactory-related genes in the antennae of Asian corn borer, Ostrinia furnacalis (Guenée) (Lepidoptera: Crambidae). PLoS One, 10: e0128550. https://doi.org/10.1371/journal. pone. 0128550

Zhang, J., Liu, Y., Walker, W.B., Dong, S.L. and Wang, G.R., 2015c. Identification and localization of two sensory neuron membrane proteins from Spodoptera litura (Lepidoptera: Noctuidae). Insect Sci., 22: 399-408. https://doi.org/10.1111/17447917.12131

Zhang, Y.N., Xia, Y.H., Zhu, J.Y., Li, S.Y. and Dong, S.L., 2014. Putative pathway of sex pheromone biosynthesis and degradation by expression patterns of genes identified from female pheromone gland and adult antenna of Sesamia inferens (Walker). $J$. chem. Ecol., 40: 439-451. https://doi.org/10.1007/ s10886-014-0433-1

Zhao, H.T., Du, Y.L., Gao, P.F., Wang, S.J., Pan, J.F. and Jiang, Y.S., 2016. Antennal transcriptome and differential expression analysis of five chemosensory gene families from the Asian honeybee Apis cerana. PLoS One, 11: e0165374. https://doi.org/10.1371/journal.pone.0165374

Zhao, H.X., Xiao, W.Y., Ji, C.H., Ren, Q., Xia, X.S. and Zhang, X.F., 2019. Candidate chemosensory genes identified from the greater wax moth, Galleria mellonella, through a transcriptomic analysis. Scient. Rep., 9: 10032. https://doi.org/10.1038/ s41598-019-46532-x 